\title{
Utilización de mantenedores de espacio en pacientes con pérdidas prematuras de dientes primarios
}

Natsibet Rivero García ${ }^{1}$

Aida Carolina Medina ${ }^{2}$

María Gabriela Martínez ${ }^{3}$

María Del Carmen Prieto ${ }^{4}$

\section{Resumen}

La pérdida prematura de dientes primarios ocurre cuando estos exfolian o son extraídos antes del momento fisiológico de recambio. Objetivo: determinar la asociación entre el uso de mantenedores de espacio y las pérdidas prematuras de dientes primarios. Materiales y métodos: se realizó el estudio descriptivo, retrospectivo, longitudinal y correlacional, de 109 registros diagnósticos de pacientes entre 3-9 años, atendidos en el Postgrado de Odontología Infantil de la UCV, excluyendo aquellos con síndromes o alteraciones sistémicas. Fue evaluado el estrato socioeconómico, dientes perdidos y el uso del mantenedor de espacio Se aplicaron pruebas estadísticas descriptivas, X2 de Pearson, y T de Student $(\mathrm{p}=0,05)$. Resultados: la edad de la muestra fue $4,93 \pm 1,5$ años. La prevalencia de pérdidas prematuras fue $70,90 \%$, la me- dia de dientes extraídos fue $3,75 \pm 2,56$. La causa más frecuente fue la caries $76,5 \%$ y el diente más afectado el 64 con 11,11\%. El 72,12\% de los pacientes utilizó mantenedor de espacio, siendo el más usado el fijo en 52,27\% No hubo asociación estadísticamente significativa entre el estrato socioeconómico con el número de dientes afectados ni con el uso o no de mantenedor de espacio. Conclusión: la prevalencia de pérdidas prematuras para este estudio fue elevada debido a las características inherentes al tipo de servicio especializado en el que se brinda atención a pacientes con requerimientos de tratamiento complejo. En la mayoría de los casos se cumplió satisfactoriamente con el uso de mantenedores de espacio como elemento de prevención de maloclusiones.

Palabras Clave: diente primario, mantenimiento del espacio.

\section{Artigo Original}

\section{Utilizaçâo de mantenedores de espaço em pacientes con perdas prematuras de dentes decíduos}

A perda prematura de dentes decíduos ocorre quando estes dentes esfoliam ou são extraídos antes do tempo da erupção fisiológica do dente permanente. Objetivo: Determinar a associação entre o uso de mantenedores de espaço e de perda prematura de dentes decíduos. Materias e

${ }^{1}$ Especialista en Odontología Infantil. Teniente de Navío, Adjunta al Área de Odontopediatria del Centro de Especialidades Odontológicas de la Armada de Venezuela.natsibetrivero@gmail.com

${ }^{2}$ Especialista en Odontología Infantil. Profesor Asociado, Departamento de Odontología Pediátrica y Ortodoncia, Universidad Central de Venezuela. caromemo@gmail.com

${ }^{3}$ Especialista en Odontología Infantil. Profesor Asistente, Departamento de Odontología Pediátrica y Ortodoncia, Universidad Central de Venezuela.

${ }^{4}$ Especialista en Odontología Infantil. Profesor Instructor. Departamento de Odontología Pediátrica y Ortodoncia, Universidad Central de Venezuela. 
método: Foi realizada uma pesquisa descritiva, registros retrospectivos, longitudinal e transversal de 109 pacientes diagnosticados entre 3-9 anos de idade, atendidas no Pós-Graduação de Odontopediatria da UCV, excluindo aqueles com síndromes ou doenças sistêmicas. O nível socioeconômico foi avaliado, falta de dentes e uso de testes de mantenedor de espaço foram aplicadas em estatísticas descritivas, X2 de Pearson e teste $t$ de Student ( $p=0,05)$. Resultados: A idade da amostra foi de 4,93 $\pm 1,5$ anos. A prevalência de perda prematura foi $70,90 \%$, a média de dentes extraídos foi de 3,75 $\pm 2,56$. A cárie foi a causa mais comum da causa das perdas com $76,5 \%$ e o dente mais ausente foi o $64 \mathrm{com}$
$11,11 \% .72,12 \%$ dos pacientes utilizou o mantenedor de espaço e o mais utilizado foi o fixo com $52,27 \%$. Não houve associação estatisticamente significante entre o nível socioeconômico com o número de dentes afetados ou o uso ou não do mantenedor de espaço. Conclusäo: A prevalência de perda prematura para este estudo foi alta, devido às características inerentes ao tipo de serviço especializado, que oferece atendimento a pacientes com necessidades de tratamento complexos. Na maioria dos casos a utilização dos mantenedores foi de forma satisfatória como recurso na prevenção de maloclusões.

Palavras-chave: dentes decíduos, mantenedores de espaço.

\section{Original article}

\section{Space manteinance after premature loss of primary molars}

\section{Abstract}

Premature loss of primary molars occurs when they are extracted before their physiologic exfoliation timing. Aim: The purpose of this study is to determine the association between premature loss of primary molars and the use of space maintainers. Materials and methods: A descriptive, retrospective, correlacional study was performed, analyzing records of 109 patients, ages 3 to 9 years old, who received dental treatment at the Pediatric Dentistry Postgraduate program UCV. Children with systemic diseases or syndromes were excluded. Socioeconomic status, extracted primary teeth and use of space maintainers were studied. Pearson's X2 and Student's T tests $(p=0,05)$ were performed. Results: Mean age was $4,93 \pm 1,5$ years-old. Prevalence of prema- ture loss of primary teeth was $70,90 \%$, with an average of $3,75 \pm 2,56$ extracted teeth per child. Dental caries was the most frequent cause for extraction $(76,5 \%)$, and the most affected tooth was the maxillary left primary firs molar (64). $72,12 \%$ of patients used space maintainers, with fixed appliances accounting for 52,27\%. No statistical significance was found between socioeconomic status, number of extracted teeth or use of space maintainer. Conclusion: High prevalence of premature loss of primary teeth was observed in this sample, belonging to a highly specialized clinic where patients with complex treatment needs are admitted. For most cases satisfactory use of space maintainers was achieved as a preventive measure to avoid oclusal disturbances.

Key words: Primary teeth, space maintainers. 


\section{Introducción}

La oclusión se establece como resultado de la interacción de factores genéticos y ambientales, los cuales varían de intensidad de acuerdo a las características individuales de cada sujeto. Entre las variables que tienen mayor influencia en el desarrollo de la oclusión dental, destaca la vulnerabilidad de la dentición primaria al ataque de la caries dental, pues ésta puede propiciar tanto la pérdida de espacio, cuando las lesiones son amplias y se encuentran ubicadas interproximalmente, como la pérdida prematura de uno o más dientes afectados. ${ }^{1,2,3,4}$

Se considera pérdida prematura de dientes primarios cuando éstos se exfolian o son extraídos antes del momento fisiológico de recambio. Sus consecuencias incluyen cambios en el plano sagital: pérdida de perímetro de arco y longitud de arco por migración mesial de los molares permanentes, y por distoinclinación o retrusión de los dientes anteriores, pérdida del espacio disponible para la correcta alineación de los dientes permanentes, lo cual producirá apiñamiento, erupción ectópica o impactación dentaria. En el plano vertical, producen profundización de la mordida y en el plano transversal, mordidas cruzadas. ${ }^{5}$

La prevalencia de pérdidas prematuras es variable de acuerdo con la población estudiada y el año en el que se haya realizado el estudio. Los reportes varían de $49,65 \%{ }^{1}$ hasta $29,4 \%{ }^{3}$

Los mantenedores de espacio son aparatos ortodóncicos que pretenden impedir el cierre de espacio, seguido de la pérdida prematura de un diente primario. Se clasifican en fijos y removibles de acuerdo a su retención. Para su colocación deben ser tomados en cuenta ciertos factores como son: la edad a la que ocurrió la pérdi- da, tipo de diente perdido, presencia del diente sucesor, análisis del espacio disponible y tiempo transcurrido desde la pérdida. Su uso es indicado por la mayoría de los autores $6,7,8,9,10$ aunque para Ngan 199611, Ghafari 1986 y Woodward 1989 citado por Brothwell $1997^{6}$ sugieren que en algunos casos pueden ser innecesario su uso.

Es necesario intervenir de manera temprana y adecuada sobre la dentición primaria para prevenir la caries, o tratar de forma conservadora los dientes con lesiones de caries, para evitar el desarrollo de una maloclusión; y en aquellos casos donde los dientes se hayan perdido prematuramente, el uso de mantenedores de espacio es indispensable para preservar la integridad del perímetro del arco dental, de las relaciones oclusales y de la dimensión vertical. ${ }^{4}$

\section{Objetivo}

Establecer la relación entre el uso de mantenedores de espacio y las pérdidas prematuras de dientes primarios en los pacientes pediátricos de 3 a 9 años atendidos del Postgrado de Odontología Infantil de la Universidad Central de Venezuela cohorte 2009-2011.

\section{Material y métodos}

Se diseño un estudio documental, descriptivo, observacional correlacional, longitudinal y retrospectivo. La población la constituyeron las historias clínicas, fotografías y radiografías de 109 pacientes pediátricos que asistieron al servicio de Clínica integral y Quirófano del postgrado de Odontología Infantil de la Universidad Central de Venezuela durante la Cohorte 2009_ 2011, las cuales fueron analizadas.

La muestra estuvo conformada por todos los pacientes que integraron la población (109), de la 
cual se excluyeron 23 pacientes quienes presentaban alguna enfermedad sistémica, anomalías congénitas o alteraciones genéticas, quedando representada por 86 pacientes de ambos géneros entre 3 y 9 años de edad. El criterio de inclusión en la muestra de estudio fue la perdida prematura de dientes primarios. Se consideró pérdida prematura cuando el diente es extraído antes del momento fisiológico de recambio tomando en cuenta la edad del paciente para el momento de la exodoncia, o de la ausencia del diente para el momento en el que se realizó el odontodiagrama.

Para los registros se tomaron los datos demográficos edad, género, estrato social y tipo de atención odontológica realizada, los cuales fueron insertados en la hoja de recolección de datos, posteriormente se evaluó el odontodiagrama, fotografías clínicas y radiografías presentes en las historias clínicas, en busca de pérdidas prematuras o dientes con indicaciones de extracción, así mismo se registró la causa de la exodoncia, tipo de diente, zona afectada, si se usó o no mantenedor de espacio, tiempo transcurrido entre la exodoncia y la instalación del mantenedor de espacio, tipo de mantenedor de espacio, número de controles realizados y perdidos al aparato y complicaciones asociadas a su uso. El estrato socioeconómico se determinó por medio del método Graffar de Mendez Castellanos, el cual divide los estratos socioeconómicos del I al V (Estrato I: clase alta, Estrato II: clase media alta, Estrato III: clase media media, Estrato IV: pobreza relativa (13 a 16 puntos), Estrato $\mathrm{V}$ : pobreza crítica). ${ }^{12}$ Se dividieron los pacientes según el tipo de atención en aquellos tratados convencionalmente en la sala clínica y los que requirieron atención bajo anestesia general.

Para el procesamiento de datos computarizado se utilizo un software estadístico versión 18 y el grado de correlación de variables fue establecido mediante el coeficiente de X2 de Pearson, y T de Student.

Este estudio es observacional, el cual se limita a describir y establecer relaciones entre fenómenos observados. No se compromete la identidad de los pacientes. El tratamiento realizado no fue manipulado por fines de investigación y cada caso contó con el consentimiento informado por parte del representante. Esta investigación recibió el aval del Comité de Bioética de la Facultad de Odontología identificado como: FO/UCV N ${ }^{\circ}$ 0196-2011.

\section{Resultados}

La edad de la muestra fue de 4,93 \pm 1,5 años, la prevalencia de perdidas prematuras fue de $70,90 \%$, la media de dientes extraídos por paciente fue de 3,75 $\pm 2,56$ de desviación estándar, del total de pacientes con perdidas prematuras encontrándose que un diente perdido por paciente fue el más frecuente con un 26,22\%.

En relación al estrato social, del total de pacientes con pérdidas prematuras, el mayor porcentaje estuvo en los estratos III $(39,53 \%)$ IV $(47,64 \%)$ y V (6,97\%). Al cuantificar la prevalencia de pérdida prematura de dientes primarios por estrato, se obtiene que la misma oscila entre $60 \%$ (estrato II) hasta 75,60\% estrato (IV). Aunque la prevalencia de pérdidas prematuras tiende a aumentar a medida que empeora el estrato socioeconómico, en este grupo no se observó una correlación estadísticamente significativa. (Fig. 1).

La distribución de los pacientes con pérdidas prematuras según el tipo de atención arrojó lo siguiente: de los 61 pacientes con pérdidas prematuras, 54 requirieron atención en sala 


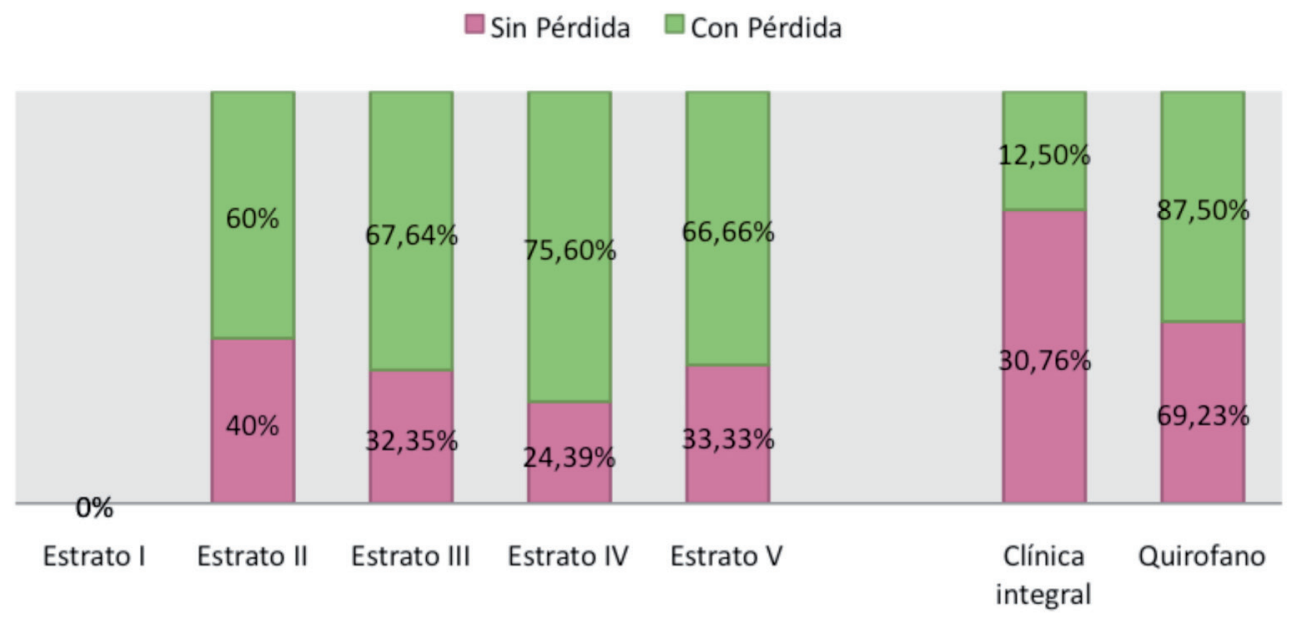

Fig. 1. Porcentaje de pacientes con pérdida prematura según el estrato social y tipo de atención.

de clínica integral representando el 88,52\% y 7 fueron atendidos bajo anestesia general lo que representó el $11,47 \%$ del total de pacientes con pérdida prematura; de igual manera se realizó el cálculo en porcentaje de pérdida prematuras del total de pacientes atendidos en cada uno de los servicios quedando establecido de la siguiente manera, en sala clínica integral del total

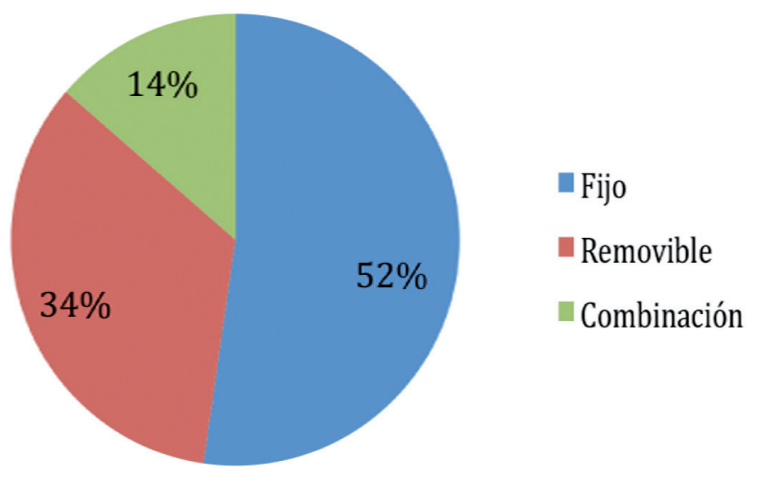

Fig. 2. Tipo de mantenedor utilizado.

Tabla 1. Frecuencia y porcentaje de dientes perdidos por paciente según tipo de atención

\begin{tabular}{|c|c|c|c|c|c|c|c|}
\hline & & \multicolumn{4}{|c|}{ Tipo de atención } & \multirow{2}{*}{\multicolumn{2}{|c|}{ Total }} \\
\hline & & \multicolumn{2}{|c|}{ Sala clínica } & \multicolumn{2}{|c|}{ Quirófano } & & \\
\hline & & Frec. & $\%$ & Frec & $\%$ & Frec & $\%$ \\
\hline \multirow{11}{*}{$\begin{array}{c}\mathrm{N}^{\circ} \text { dientes } \\
\text { perdido por } \\
\text { paciente }\end{array}$} & 1 & 15 & $24,59 \%$ & 1 & $1,63 \%$ & 16 & $26,22 \%$ \\
\hline & 2 & 8 & $13.11 \%$ & 0 & $0 \%$ & 8 & $13,11 \%$ \\
\hline & 3 & 9 & $14,75 \%$ & 1 & $1,63 \%$ & 10 & $16,39 \%$ \\
\hline & 4 & 6 & $9,83 \%$ & 0 & $0 \%$ & 6 & $9,83 \%$ \\
\hline & 5 & 5 & $8,19 \%$ & 1 & $1,63 \%$ & 6 & $9,83 \%$ \\
\hline & 6 & 5 & $8,19 \%$ & 2 & $3,27 \%$ & 7 & $11,46 \%$ \\
\hline & 7 & 4 & $6,55 \%$ & 0 & $0 \%$ & 4 & $6,55 \%$ \\
\hline & 8 & 1 & $1,63 \%$ & 0 & $0 \%$ & 1 & $1,63 \%$ \\
\hline & 9 & 1 & $1,63 \%$ & 0 & $0 \%$ & 1 & $1,63 \%$ \\
\hline & 10 & 0 & $0 \%$ & 1 & $1,63 \%$ & 1 & $1,63 \%$ \\
\hline & 13 & 0 & $0 \%$ & 1 & $1,63 \%$ & 1 & $1,63 \%$ \\
\hline \multicolumn{2}{|l|}{ Total } & 54 & $88,47 \%$ & 7 & $11,42 \%$ & 61 & $100 \%$ \\
\hline
\end{tabular}


Tabla 2. Frecuencia y distribución de los pacientes según el uso de mantenedores de espacio y lugar de atención.

\begin{tabular}{|c|c|c|c|c|c|}
\hline \multicolumn{2}{|c|}{} & \multicolumn{4}{c|}{ Lugar de atención } \\
\cline { 3 - 6 } \multicolumn{2}{|c|}{} & \multicolumn{2}{|c|}{ Sala Clínica } & \multicolumn{2}{c|}{ Quirófano } \\
\cline { 3 - 6 } & Frecuencia & Porcentaje & Frecuencia & Porcentaje \\
\hline \multirow{2}{*}{$\begin{array}{c}\text { Uso de } \\
\text { mantenedor }\end{array}$} & No uso & 12 & $22,22 \%$ & 5 & $71,42 \%$ \\
\cline { 2 - 6 } & Si uso & 42 & $77,77 \%$ & 2 & $28,57 \%$ \\
\hline \multicolumn{2}{|c|}{ Total } & 54 & $100 \%$ & 7 & $100 \%$ \\
\hline
\end{tabular}

de pacientes sanos atendidos (78) 54 pacientes presentaron pérdidas prematuras representando el 69,23\% y del total de pacientes sanos atendidos en quirófano (8) 7 presentaron pérdidas prematuras representando un $87,5 \%$ del total de pacientes que recibieron atención en quirófano. (Fig. 1).

De igual forma se realizó una distribución por frecuencia y porcentaje según el tipo de atención, hallando que de los 54 pacientes con pérdida prematura atendidos en sala clínica, 15 pacientes presentaron pérdida de un diente, siendo el más frecuente con el 24,59\%, y en quirófano de los 7 pacientes atendidos, 2 presentaron pérdida de seis dientes primarios siendo el más frecuente con 3,27\% del total de pacientes. Así mismo se halló que la media de dientes perdidos por paciente en sala de clínica integral fue de 3,37 $\pm 2,20$ de desviación estándar, y en quirófano la media de dientes perdidos por paciente fue de $5,62 \pm 3,93$ de desviación estándar.

De lo observado clínicamente hubo una correlación positiva estadísticamente significativa (X2 Pearson $\quad \mathrm{p} \leq 0,01)$ entre el número de dientes perdidos por paciente y el tipo de tratamiento requerido, siendo para el grupo de pacientes atendidos en sala clínica el número máximo de perdidas prematuras por paciente de 9 , a pesar de estar conformado por un número mayor de pacientes comparado con el grupo de pacientes de quirófano en el cual se presentó un máximo de 13 perdidas en un paciente. De esto se deriva que los pacientes que requieren tratamiento bajo anestesia general tienen una probabilidad mayor de requerir exodoncias múltiples que los pacientes atendidos en sala clínica.

Para la Prueba T Student $(\mathrm{p}=0,05)$ no hubo diferencias estadísticamente significativas del $\mathrm{N}^{\mathrm{o}}$ de dientes perdidos por paciente de clínica integral y quirófano. Así, aunque los pacientes atendidos en quirófano tienen requerimiento de mayor número de exodoncias, la media de dientes perdidos no difiere, de forma estadísticamente significativa, entre ambos grupos.

Con respecto al tipo de diente perdido, el 64 (primer molar primario superior izquierdo) presentó la mayor frecuencia, representando 25 dientes de los 226 dientes perdidos con un porcentaje de 11,1\%. (Tabla 3).

Respecto a la localización y número de dientes perdidos agrupados en Incisivos, caninos, primeros molares primarios y segundos molares primarios, en el maxilar la mayor frecuencia la presentó el grupo de los incisivos con 67 dientes perdidos, representando el $29,64 \%$ del total de dientes perdidos, seguido por el grupo de los primeros molares primarios con 44 dientes 
perdidos para un 19,46\%, y en la mandíbula la didos, representado el 19,02\% del total, seguido mayor frecuencia la presentó el grupo de los por el grupo de los segundos molares primarios primeros molares primarios, con 43 dientes per- $\quad$ con 36 dientes perdidos representando el 15,92\%

Tabla 3. Dientes primarios extraídos y causa de la extracción.

\begin{tabular}{|c|c|c|c|c|c|}
\hline \multirow{2}{*}{ Diente } & \multicolumn{4}{|c|}{ Causa } & \multirow{2}{*}{ Total } \\
\hline & Desconocida & Caries & Traumatismo & Otra & \\
\hline 51 & 4 & 15 & 2 & - & 21 \\
\hline 52 & - & 12 & 1 & - & 13 \\
\hline 61 & 5 & 15 & - & - & 20 \\
\hline 62 & 1 & 12 & - & - & 13 \\
\hline 71 & - & - & - & - & 0 \\
\hline 72 & - & - & - & 1 & 1 \\
\hline 81 & - & - & - & - & 0 \\
\hline 82 & - & - & - & 1 & 1 \\
\hline Total incisivos & 10 & 54 & 3 & 2 & 69 \\
\hline $31 \%$ & $14 \%$ & $78 \%$ & $4 \%$ & $3 \%$ & \\
\hline 53 & - & 1 & 1 & 1 & 3 \\
\hline 63 & - & 2 & - & 1 & 3 \\
\hline 73 & - & 1 & - & 2 & 3 \\
\hline 83 & - & 1 & - & 2 & 3 \\
\hline Total caninos & 0 & 5 & 1 & 6 & 12 \\
\hline $5 \%$ & $0 \%$ & $42 \%$ & $8 \%$ & $50 \%$ & \\
\hline 54 & 3 & 16 & - & - & 19 \\
\hline 55 & 1 & 8 & - & - & 9 \\
\hline 64 & 4 & 21 & - & - & 25 \\
\hline 65 & 1 & 12 & - & - & 13 \\
\hline 74 & 5 & 16 & - & - & 21 \\
\hline 75 & 4 & 11 & - & 1 & 16 \\
\hline 84 & 5 & 17 & - & - & 22 \\
\hline 85 & 7 & 13 & - & - & 20 \\
\hline Total molares & 30 & 114 & 0 & 1 & 145 \\
\hline $64 \%$ & $21 \%$ & $79 \%$ & $0 \%$ & $1 \%$ & \\
\hline Total general & 40 & 173 & 4 & 9 & 226 \\
\hline $100 \%$ & $18 \%$ & $77 \%$ & $2 \%$ & $4 \%$ & \\
\hline
\end{tabular}


Tabla 4. Frecuencia de pacientes según el estrato social, lugar de atención y uso o no de mantenedores de espacio.

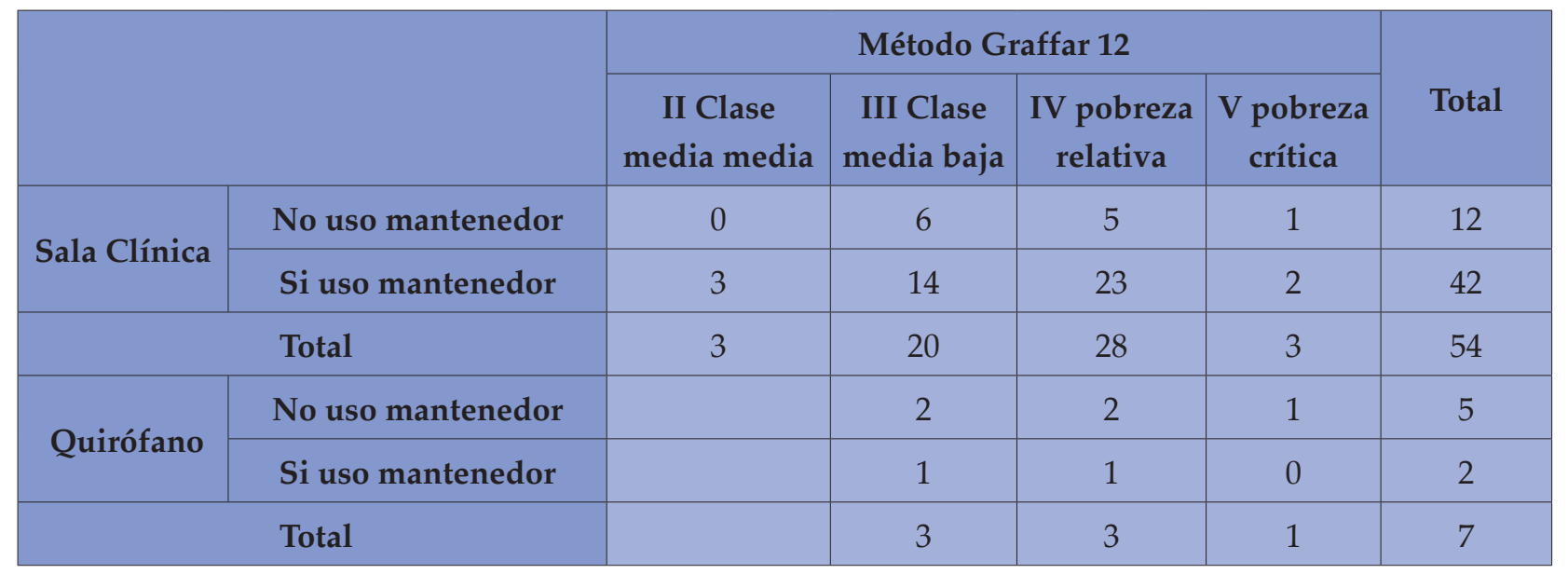

del total. Respecto a lo anterior se pudo observar que la zona más afectada por las pérdidas prematuras es la zona media en un $69,5 \%$ con respecto a la anterior con un $30,5 \%$. (Tabla 3 ).

En relación a la causa de la pérdida prematura la mayor frecuencia, tanto para los dientes del segmento anterior como para molares, fue la caries, afectando a 173 de los 226 dientes perdidos con un porcentaje de 76,5\%. (Tabla 3).

Con respecto al momento en el que ocurrió la pérdida prematura se pudo observar que de los 226 dientes perdidos, 177 fueron extraídos durante el tratamiento representando el 78,3\% y a los $49(21,7 \%)$ dientes restantes ya se les había realizado la exodoncia, al momento de realizar la primera evaluación para la historia clínica. Asimismo, se determinó que la media del tiempo transcurrido entre la exodoncia y la instalación del mantenedor de espacio fue de 3 meses y 8 días $\pm 0,23$ de desviación estándar.

En relación a la terapéutica aplicada para el tratamiento de las pérdidas prematuras, en sala clínica integral de los 54 pacientes con pérdida 42 recibieron mantenedores de espacio representando un 77,77\% y 12 pacientes, no usaron mantenedores debido a erupción del diente permanente, razones económicas o el paciente abandonó el tratamiento. Asimismo, en quirófano de los 7 pacientes con pérdidas prematuras de dientes primarios, 2 recibieron mantenedores de espacio representado el 28,57\%, y los 5 pacientes restantes $71,42 \%$ no recibieron ningún tipo de terapéutica por las razones antes referidas. Con respecto a lo encontrado clínicamente, existe una correlación estadísticamente, ligera e inversa de las variables y es significativa con la prueba X2 ( $<<0,01)$, ya que los pacientes que fueron atendidos en sala de clínica integral fueron más los que recibieron mantenedores de espacio respecto a los que no, a diferencia de los pacientes de quirófano que fueron más los que no recibieron alguna terapéutica para las perdidas prematuras que aquellos que si la obtuvieron. Los pacientes que requirieron tratamiento bajo anestesia general tienen probabilidad de no utilizar los mantenedores de espacio.

Relativo a la relación que existe entre el estrato social y el uso o no de mantenedores de espacio tanto en sala clínica integral como quirófano, no se encontró para esta muestra una diferencia estadísticamente significativa, sin embargo, se 
reportó una correlación inversa $(-0,52)$ es decir, a medida que aumenta el Graffar (estrato social bajo), disminuye el uso de mantenedores de espacio. (Tabla 4).

Con respecto al tipo de mantenedor de espacio usado, en sala clínica integral el más frecuente fue el fijo con un $52,38 \%$, seguido por el removible con $33,33 \%$ y el combinado con un $14,28 \%$, asimismo en quirófano se obtuvo la misma frecuencia con 1 fijo y 1 removible representando el $50 \%$ cada uno.

Respecto al número de controles realizados a los mantenedores de espacio la mayor frecuencia la obtuvo el de 3 controles con un porcentaje de $36,36 \%$.

Con respecto a si los pacientes tuvieron o no complicaciones con el aparato, del total de paciente (44) solo 11 presentaron algún tipo de complicación siendo la de mayor frecuencia el desalojo, presentándose en 4 pacientes lo cual representó el 9,09\%. Relacionando el tipo de mantenedor con las complicaciones presentes se pudo observar que hubo más complicaciones en los pacientes con mantenedores fijos que los que usaron removible.

\section{Discusión}

En el Postgrado de Odontología Infantil de la UCV se brinda atención odontológica integral a los pacientes pediátricos, los cuales son seleccionados previamente a través de un triaje, cuyas necesidades terapéuticas son complejas, por lo que ameritan tratamiento especializado. El servicio tiene finalidad docente y asistencial, al respecto el tipo de atención requerida por el paciente varía de acuerdo a las características propias del tratamiento a realizar, y del paciente. Es así como los pacientes de corta edad, difícil comportamiento y tratamientos odontológico extensos y complejos son atendidos bajo anestesia general, y aquellos que tienen más de 3 años y que ameriten tratamiento integral serán atendidos en sala de clínica integral del Postgrado.

Es importante resaltar que todos los pacientes tienen alta prevalencia de caries para ingresar al servicio, de allí la gran cantidad de exodoncias indicadas, por el avance de la lesión o por la imposibilidad de realizar un tratamiento restaurador que garantice su permanencia en boca. Cabe destacar que antes de tomar la decisión de realizar la exodoncia se han considerado las diferentes opciones de tratamiento (preventivo, endodóntico y restaurador) para mantener al diente. Para esta investigación la prevalencia de pérdidas prematuras fue alta con un $70,9 \%$ con una media de dientes perdidos por paciente de 3,75 $\pm 2,56$. Estos resultados no son comparables con la población general, ni con otros estudios realizados en poblaciones ortodoncias, ya que es una población sesgada con alta prevalencia en caries. Cuando se comparó el número de dientes perdidos por paciente con el tipo de atención requerida, se halló una correlación estadísticamente significativa siendo para el grupo de pacientes atendidos en sala clínica el número máximo de perdidas prematuras por paciente de 9 , a pesar de estar conformado por un número mayor de pacientes comparado con el grupo de pacientes de quirófano en el cual se presentó un máximo de 13 perdidas en un paciente. De esto se deriva que los pacientes que requieren tratamiento bajo anestesia general, tienen una mayor probabilidad de requerir exodoncias múltiples ya que el tratamiento es más radical, tanto por la necesidad del paciente, como por la imposibilidad de luego continuar con tratamiento ambulatorio, por lo que el tratamiento debe ser realizado todo en el mismo acto quirúrgico. Asimismo cuando 
se compararon las medias de dientes perdidos en sala clínica $(3,37 \pm 2,20)$ y en quirófano $(5,62$ $\pm 3,93$ ) se pudo apreciar que fue mayor en quirófano, sin embargo no hubo diferencia estadísticamente significativa entre los dos grupos, lo cual puede ser debido a lo reducido del tamaño de la muestra.

Al comparar el número de exodoncias con el estrato social clínicamente, se pudo apreciar una asociación con las pérdidas prematuras es decir, aquellos pacientes con un estrato social bajo (IV) presentaron una tendencia hacia un mayor número de perdidas prematuras, en comparación con aquellos que tenían un estrato más alto, sin embargo, esta asociación no fue estadísticamente significativa. Esto puede relacionarse con los patrones de caries según el estrato social y la inequidad en atención odontológica para estratos más pobres, similar a lo reportado por FUNDACREDESA. ${ }^{12}$

Con respecto a la frecuencia de la cantidad de dientes perdidos por paciente lo más común fue de un diente perdido por paciente coincidiendo con Pedersen. ${ }^{1}$

Al analizar las causas de las perdidas prematuras, se encontró que la más frecuente fue la caries con un 76,5 esto es similar a lo reportado por Pedersen y $\mathrm{Col}^{1}$, Ortiz y $\mathrm{col}^{13}$, Cardozo y $\operatorname{col}^{14}$ y Lin y col. ${ }^{15}$ De aquí la importancia de instaurar medidas preventivas y restauradoras en etapas tempranas, considerando que es el principal agente etiológico de las pérdidas prematuras. También fueron halladas otras causas de pérdidas prematuras como los traumatismos los cuales ocuparon la menor proporción la pérdida de caninos inferiores y de incisivos laterales inferiores estuvieron relacionadas principalmente con discrepancia negativa coincidiendo con Ngan y col y Van Der Linden ${ }^{11,16}$. Hay casos en los cuales la causa no estuvo claramente definida en la historia clínica, y otros en los cuales los pacientes ya presentaban exodoncias previas al momento de realizar la historia clínica, por lo que se desconoce la causa de la pérdida.

Según lo reportado por Fanning ${ }^{17}$ Brin y $\operatorname{cols}^{18}$, respecto a las edades medias, en las distintas etapas de formación radicular de los dientes sucesores, y compararlas con la edad media de este estudio que fue de $4,93 \pm 1,5$, se puede inferir que para esta edad que es antes de los 5 años aún no se ha completado la primera etapa de formación radicular lo cual llevara posiblemente a un retardo de la erupción de los dientes sucesores.

La mayoría de los pacientes con pérdidas prematuras utilizaron mantenedores de espacio en un $72,13 \%$. Es importante señalar que en el tratamiento en el postgrado se hace énfasis en lograr rehabilitación integral, incluyendo mantenedores de espacio.

Según Lin y cols ${ }^{15}$ la mayor migración mesial, ocurre hasta los 6 meses después de haber realizado la exodoncia, siendo este período crítico para lograr evitar la pérdida del perímetro del arco. Respecto a lo antes descrito se constató que el tiempo medio transcurrido entre la exodoncia y la colocación del mantenedor de espacio fue de 3 meses y 8 días \pm 2 meses y 24 días. Este lapso de tiempo se ubica en el que se producen los mayores cambios de pérdida de espacio. Sin embargo pudiera tratar de disminuirse el tiempo y colocarlos más temprano.

Los pacientes que requirieron tratamiento en quirófano, en su mayoría no usaron mantenedor de espacio. La diferencia entre el uso de mantenedor en ambos grupos (sala clínica y quirófano) fue estadísticamente significativa, 
siendo los que presentaron menor probabilidad de utilizar mantenedores los pacientes que fueron atendidos bajo anestesia general. Entonces, el paciente que requiere anestesia general tiene mayor probabilidad de tener pérdidas prematuras múltiples, y menor probabilidad de utilizar mantenedor de espacio, lo cual puede deberse a que son pacientes que presentaron una media de edad menor, o que su inhabilidad para colaborar con el tratamiento imposibilita la colocación del mantenedor. De allí la importancia de realizar sensibilización a los padres para que no abandonen el tratamiento y continúen con la colocación de mantenedores.

El tipo de mantenedor de espacio más frecuentemente utilizado fue el fijo. Este tipo de mantenedor no requiere de la colaboración del paciente en cuanto a su remoción o inserción en casa, sin embargo requiere normas de higiene adecuadas.

El mantenedor de espacio fijo también fue el que presentó mayor cantidad de complicaciones. En general, el 75\% de los pacientes no presentó ningún tipo de complicación con el uso de los mantenedores, tanto fijos como removibles. Esto concuerda con el estudio realizado por Rodd y $\mathrm{col}^{19}$ quienes midieron la frecuencia de reparaciones en prótesis parcial removible superior para el reemplazo de un incisivo permanente, obteniendo una frecuencia de $36,2 \%$. De igual manera en el cuestionario aplicado a los niños, indicó un nivel muy alto de molestia percibido por los pacientes portadores de la prótesis y la cual aumentaba con el tiempo.

El uso de mantenedores de espacio requiere controles periódicos. En la historia clínica sólo fueron registradas pocas citas de control con inasistencia del paciente, no pudiendo evaluarse de forma eficiente la adherencia al tratamiento.
Sí fueron anotadas las citas en las que el paciente eficazmente acudió a control. El $61 \%$ acudió de 3 a 4 citas y sólo el $6 \%$ logró cumplir con 6 a 8 citas. Esto puede ser debido a que se evaluó en un período de 2 años como máximo de seguimiento, habiendo pacientes que comenzaron sus tratamientos ya cercanos a la fecha de culminación de la cohorte, siendo imposible haber cumplido tres controles. Pero esto puede deberse a que el mínimo indispensable desde el punto de vista administrativo, que debe cumplir el paciente es 3 controles, disminuyendo la adhesión al tratamiento después de haber cumplido estos.

El tratamiento odontológico integral del paciente pediátrico debe realizarse bajo una planificación acorde con la edad y la complejidad del caso. Cuando el paciente presenta múltiples lesiones de caries con afección pulpar e imposibilidad de restauración, se hace necesaria la realización de exodoncias. En este estudio se evidencia elevada prevalencia de pérdidas prematuras, alcanzando 70,9\%. Cabe destacar que un elevado porcentaje de pacientes, utilizó los mantenedores de espacio ampliamente descritos en la literatura. (6.10) Por ser este un servicio especializado con fines docentes y asistenciales se hace especial énfasis en lograr cumplir con todos los aspectos del tratamiento integral, incluyendo uso de mantenedores de espacio.

Para lograr el éxito del tratamiento es indispensable la adhesión del representante y del niño al mismo, por lo cual es necesaria una adecuada motivación y educación por parte del odontopediatra para mantener un buen estado de salud bucal.

\section{Conclusiones}

En los pacientes sanos atendidos en clínica integral y quirófano en el Postgrado de Odonto- 
logía Infantil entre el 2009-2011, la prevalencia de pérdidas prematuras fue $70,90 \%$, mostrando predilección por el género masculino, en edades comprendidas de 4 a 5 años y pertenecientes en su mayoría a un estrato social de IV y III.

La etiología de las pérdidas prematuras fue la caries $76,5 \%$. El diente y la zona más afectada por la pérdida fueron el diente 64 con 11,11\%, y la zona media con el $69,5 \%$. El promedio de dientes perdidos por paciente fue de 3,75 $\pm 2,56$, y la mayoría presentó pérdida de un diente.

El 72,12\% de los pacientes con pérdidas prematuras usaron mantenedor de espacio, siendo el fijo el tipo de mantenedor más frecuente y el que más complicaciones presentó, (fractura y lesión de los tejidos blandos) El número de controles promedio realizados al mantenedor de espacio, fue de 3 .

La atención odontológica del paciente pediátrico incluye prevención, restauración y en los ca- sos más complejos exodoncias, las cuales deben ir acompañadas del uso de mantenedores de espacio, si las mismas fueron realizadas prematuramente. De allí la importancia del Odontopediatra y de su intervención temprana a fin de garantizar en el niño óptimas condiciones de salud bucal.

\section{Recomendaciones}

Se enfatiza en la importancia de la prevención de caries para preservar el correcto estado de salud bucal. Debe lograrse la motivación de los representantes y los Odontólogos en el uso de mantenedores de espacio, para lo casos en los que ocurra pérdida prematura de múltiples dientes primarios. No sólo en servicios especializados, sino en todos los casos (públicos, servicio social, etc) lo cual disminuiría el riesgo a desarrollar una maloclusión, disminuyendo a su vez la necesidad de tratamientos más complejos con reducción de costos.

\section{Referencias}

1. Pedersen J, Stensgaard K, Melsen B. Prevalence of malocclusion in relation to premature loss of primary teeth. Community Dent Oral Epidemiol 1978, 6: 204-209.

2. Medrano J, Celdillo L, Murrieta J. Prevalencia de factores de riesgo para el desarrollo de la oclusión. Rev. ADM, 2002; LIX (4) 128-133.

3. Karaiskos N, Wiltshire W, Odlum O, Brothwell D, Hassrd T. Prevenive and Interceptive Orthodontic Treatment Needs o fan Inner-City Group of 6 -and 9 year Old Canadian Children. JCDA, 2005; 71, (9): 649-649e.

4. Medina A, Crespo O, Da Silva L. Factores de riesgo asociados a maloclusión en pacientes pediátricos. Acta Odont. Venez. 2010; 48.(2) 1-13

5. García Y, Da Silva L, Medina A, Crespo O. Efecto de la pérdida prematura de molares primarios sobre la relación horizontal incisiva. Rev. Odontopediatr Latinoam. 2011; 1(1): 49-57.

6. Brothwell, D. Guidelines on the use of space maintainers following premature loss of primary teeth. J Res Dent 1997; 63, (10): 753-766.

7. Terlaje R, Donly K. Treatment planning for space maintenance in the primary and mixed dentition. J Dent Child 2001; 68 (2) :109-114.

8. Bijoor R, Kohli K. Comtemporary Space Maintenance. J Paediatr Dent. 2005; 32-35.

9. Laing E, Ashley P, Naini F, Gill D. Space maintenance. Int J Paediatr Dent. 2009;19:155-162. 
10. Ram D, Ben M, Efrat J, Moskovitz M. Mantenedores de espacio mandibulares para la prevención de pérdida de espacio, posterior a la pérdida prematura de molares primarios. Odontol Pediatr. 2010; 9(2):127-140.

11. Ngan P, Alkire R, Fields H. Management of space problems in the primary and mixed dentitions. J Am Dent Assoc, $1999 ; 130,1330-1339$.

12. FUNDACREDESA. La Caries en la Dentición Primaria. En Estudio Nacional de Crecimiento y Desarrollo Humano de la República de Venezuela. “Proyecto Venezuela”. Caracas 1986.

13. Ortiz M, Godoy S, Farias M, Mata M. Pérdida prematura de dientes temporales en pacientes de 5 a 8 años de edad asistidos en la Clínica de Odontopediatria de la Universidad Gran Mariscal de Ayacucho, 2004-2005 2009. Acta Odont Venez [serie en internet] [citado el 17/06/11]. Disponible en: http: / / www.ortodoncia.ws/publicaciones/2009/art17.asp.

14. Cardozo L, Zembrusqui C, Sartori D, Boff I, Pessin V. Avalicäo da prevalëncia de perdas precoces de molares decíduos. 001, Pesq Bras em Odontoped Clín Integr.Joao Pessoa. 2005 ;5: (1): 17-22.

15. Lin YT, Lin WH, Tzer Y, Lin YT. Immediate and six month space changes after premature loss of primary maxillary first molar. J Am Dent Assoc. 2007;138: 362-368.

16. Van Der Linden, Frank P.G.M. Transition of the Human Dentition. Michigan : s.n., 1982. Vol. 13.

17. Fanning E. Effect of Extraction of deciduous molars on the formation and eruption of their successors. Angle Orthod.1962; 32(1): 44-53.

18. Brin L, y Koyoumdijsky K. The Influence of premature extractions of primary molars on the ultimate root length of their permanent successors. J Dent Res. 1981; 60, 962-5.

19. Rood H, Atkin J. Denture satisfaccion and clinical performance in a paediatric population. J Int Paeditric Dent. 2000; $27-37$.

Recibido: 12-11-12

Aceptado: 29-12-12

Correspondencia: natsibetrivero@gmail.com 